\title{
LARGE DEFORMATION FINITE ELEMENT ANALYSIS OF UNDRAINED PILE INSTALLATION
}

\author{
JAKUB KONKOL, LECH BAŁACHOWSKI \\ Department of Geotechnics, Geology and Marine Civil Engineering, Faculty of Civil and Environmental Engineering, \\ Gdańsk University of Technology (GUT), Gdańsk, Poland, e-mail: jakub.konkol@pg.gda.pl, abal@pg.gda.pl
}

\begin{abstract}
In this paper, a numerical undrained analysis of pile jacking into the subsoil using Abaqus software suit has been presented. Two different approaches, including traditional Finite Element Method (FEM) and Arbitrary Lagrangian-Eulerian (ALE) formulation, were tested. In the first method, the soil was modelled as a two-phase medium and effective stress analysis was performed. In the second one (ALE), a single-phase medium was assumed and total stress analysis was carried out. The fitting between effective stress parameters and total stress parameters has been presented and both solutions have been compared. The results, discussion and verification of numerical analyzes have been introduced. Possible applications and limitations of large deformation modelling techniques have been explained.
\end{abstract}

Key words: Abaqus, Arbitrary Lagrangian-Eulerian, ALE, FEM, Pile jacking, Pile installation, Undrained analysis

\section{INTRODUCTION}

In the last few years, a rapid development of IT branch has opened up a new range of possibilities in geotechnical numerical modelling. Although the major part of methods and theory has been established in the last decades, the effective application of its capabilities becomes possible nowadays. Arbitrary Lagrangian-Eulerian (ALE) method (Donea et al., 2004) as well as Coupled Eulerian-Lagrangian (CEL) method (Noh, 1963) have been developed especially for large deformation problems and have found a practical application in modelling pile penetration problems. The main research area is related to the pile set-up in sands (e.g., Hamann et. al., 2015). Numerical modelling of penetration problems in clays is usually linked to offshore structures (e.g., Tho et al., 2013).

Installation effects due to the pile set-up are in interest of geotechnical engineers since the early $1950 \mathrm{~s}$ (e.g., Cummings et al., 1950). The main reason for that is to use the pile capacity increase due to installation in design. For clayey soils the pile capacity increase is related to the changes in stress state during installation and following consolidation phase. The ageing effects in cohesive soils play minor role (Komurka et al., 2003). The basic problem in numerical modelling of such phenomenon is naturally related with large deformations of the soil structure and at the pile-soil interface. Consequently, the application of the traditional finite element method (FEM) could be troublesome because of mesh distortions. Numerical methods for large deformation problems such as ALE or CEL are developed in frames of explicit formulation, which gives the best effectiveness. The consolidation process cannot be modelled in explicit formulation. However, it is easy to obtain with implicit formulation. Different nature of installation and consolidation processes causes extremely small amount of published FEM studies. Some strategies have been developed to solve that problem. The first one is to use both solvers. In that proposition explicit calculations are made for installation phase. Then, the entire solution is mapped by an external algorithm to the new mesh and the problem is calculated by implicit solver. This kind of analysis was done by Yi et al. (2014). Modelling pile installation and consolidation directly by implicit solver is possible, but requires special care. Such analyses were done by Zhou et al. (2013) for clayey soil and by Hamann et al. (2015) for sands.

In this paper, the possibility of pile undrained installation using an implicit solver is presented. A twophase soil model is used and calculations are performed in accordance with effective stress approach. The accuracy of the effective stress solution is controlled by independent calculation in the total stress analysis with ALE method, where explicit solver is used. Finally, the interpretation of the results with 
further possibilities of this modelling technique is discussed.

\section{PROPOSED MODELLING TECHNIQUE FOR THE PILE UNDRAINED INSTALLATION}

\subsection{IMPLICIT \\ VERSUS EXPLICIT ANALYSIS}

The modelling technique presented in this paper consists of two branches. The first one is the total stress analysis using ALE method and explicit solver. The second one is the effective stress approach, where the soil is modelled as two-phase medium and the implicit solver is used. The difference between explicit and implicit methods lies in the mathematical formulation of the problem. Let us assume the current configuration of the system $Y(t+\Delta t)$ at time $t+\Delta t$ and the previous configuration of the system $Y(t)$ at time $t$, as is shown in Fig. 1. The explicit methods calculate the state of the system at time $t+\Delta t$ from state of the system at time $t$, which can be written by the equation

$$
Y(t+\Delta t)=F(Y(t)) .
$$

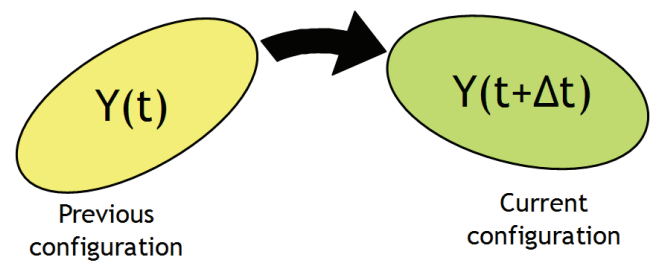

Fig. 1. Configurations of the system at two different times (e.g., during deformation of the body)

The implicit methods use different strategy. The state of the system $Y(t+\Delta t)$ is calculated from both states, at times $t$ and $t+\Delta t$, respectively. This can be written as follows

$$
F(Y(t), Y(t+\Delta t))=0 .
$$

Here, the basic advantages and disadvantages of both methods appear. The explicit method, as it is implemented in Abaqus, uses the central-difference integration rule and very small time steps defined as (Dassault Systémes, 2013)

$$
\Delta t \approx \frac{l_{e, \min }}{c_{d}}
$$

where $\Delta t$ - time increment [s], $c_{d}$ - dilatational wave speed $[\mathrm{m} / \mathrm{s}], l_{e, \min }-$ smallest element dimension in the mesh $[\mathrm{m}]$.

The features of the explicit method are very attractive and allow the large jobs to be modelled with much smaller amount of physical memory than it is required in the implicit formulation. Large processing power is needed instead. Explicit method is suitable for modelling quasi-static and dynamic events, while implicit method is perfect for calculating the static response of the system.

\subsection{ARBITRARY \\ LAGRANGIAN-EULERIAN METHOD}

Arbitrary Lagrangian-Eulerian method combines best features of Eulerian and Lagrangian formulations (Donea et al., 2004). Both soil and pile are discretized with the Lagrangian mesh. ALE description consists of three domains - the material, the spatial and the referential one. This assumption allows an arbitrary movement to be introduced between material points and the spatial (nodes) mesh. As a result, three steps of ALE description during single time increment can be specified. In the first step, the material nodes are moved to the new positions. Then, in the rezone step, a new spatial mesh is built for the best matching to the material nodes. Finally, the solution is transferred from the old mesh to the new one. As can be noticed, the crucial aspect in ALE formulation is the accuracy of remeshing algorithm. However, this method requires the same material properties in all ALE domains. Consequently, the same undrained shear strength of the soil as well as elastic parameters need to be used. This problem was reported by Bienen et al. (2015) and was also experienced by the authors in this particular study.

\subsection{COUPLED PORE FLUID DIFFUSION AND STRESS ANALYSIS BY FEM}

The coupled pore fluid diffusion and stress analysis enables us to consider the soil as a two-phase medium. This kind of modelling is especially dedicated to consolidation problems, but it can also be successfully used in undrained analysis. The framework of this formulation consists of porous elastic model combined with cap plasticity model. The coupled pore fluid diffusion and stress analysis is not intended to model the large deformation problems because of mesh distortion that may occur. The accuracy of the 
solution is controlled by the allowable pore fluid pressure in each time increment. The complementary criterion is the minimum useable time increment $\Delta t$. This value can be estimated by Vermeer and Verruijt (1981) formula for one dimensional consolidation problem

$$
\Delta t \geq \frac{\gamma_{w} \cdot\left(l_{e}\right)^{2}}{6 \cdot E \cdot k}
$$

where $\gamma_{w}$ - unit weight of water $\left[\mathrm{kN} / \mathrm{m}^{3}\right], l_{e}$ - element length $[\mathrm{m}], E$ - elastic modulus [kPa], $k$ - coefficient of permeability $[\mathrm{m} / \mathrm{s}]$.

Choosing the appropriate minimum time increment is crucial for the solution without "overshoot" in pore fluid pressure calculation. On the other hand, equation (4) is derived only for one dimensional consolidation problem, but it is also widely used in three dimensional models (e.g., Dai and Qin, 2013).

Summing up, the penetration problems calculated in terms of the coupled pore fluid diffusion and stress analysis cannot be always possible because of model geometry, mesh distortion or allowable time increment. Further, even when such analysis is possible, the obtained results may be false. These are the reasons why ALE method was used to verify coupled pore fluid diffusion and stress analysis in the pile installation problem.

\subsection{GENERAL CONSIDERATION ABOUT THIS RESEARCH}

In this paper, ALE method is considered as a major modelling technique. This implies the use of single-phase uniform material, as was explained in Section 2.2. To achieve this assumption the Tresca model with linear elastic parameters was used. For coupled pore fluid diffusion and stress analysis the Modified Cam-Clay (MCC) model was assumed. The fitting between these two models is presented in the next section. The minimum usable time increment described by equation (4) was calculated by selecting the mesh size and the coefficient of permeability.

Another problem with the coupled pore fluid diffusion and stress analysis is the modelling of soil-pile interface. Preliminary analysis of the pile jacking has shown that the Coulomb friction model cannot be applied on the pile toe. Hence, friction model on the pile shaft was assumed, while frictionless behaviour on pile toe was applied. The same interface conditions in ALE model were used.

\section{EFFECTIVE STRESS VERSUS TOTAL STRESS ANALYSIS}

In presented modelling technique the effective stress and total stress analysis will be introduced. In both approaches different constitutive models are used. To obtain reliable solutions, some correlation between Tresca parameters and MCC parameters has to be assumed. A relation between the linear elastic model and the porous elastic model is also needed. The fitting between the total stress analysis and the effective stress analysis will be performed in the following way. Firstly, the parameters for ALE model will be assumed. They are presented in Table 1. Next, the soil parameters required in the effective stress analysis will match those occurring in ALE model. To obtain correlation between the undrained and drained elastic strength parameters the assumption of equal shear modulus will be made. This can be done for the materials for which the shear and volumetric effects are decoupled (Atkinson, 2007)

$$
G^{\prime}=G_{u}=\frac{E_{u}}{2\left(1+v_{u}\right)}
$$

where $G^{\prime}$ - effective shear modulus $[\mathrm{kPa}], G_{u}$ - undrained shear modulus $[\mathrm{kPa}], E_{u}$ - undrained elastic modulus $[\mathrm{kPa}], v_{u}$ - undrained Poisson ratio [-].

Hence, the porous elastic medium in effective stress analysis can be defined by constant shear modulus $G^{\prime}$ and logarithmic elastic modulus $\kappa$. This definition of porous material will be consistent with the assumption of decoupled shear and volumetric effects (Dassault Systémes, 2013).

Another problem lies in fitting the undrained shear strength of soil $c_{u}$ to the MCC plasticity parameters. Some proposition to deal with that issue has been put forward by Potts and Zdravkovic (1999). As different parameters are used in Potts and Zdravković proposition and in Abaqus formulation, the independent derivation of the MCC parameters is made here.

Table 1. Material parameters for ALE model (Linear elastic with Tresca plasticity)

\begin{tabular}{|c|c|c|c|c|c|c|c|c|}
\hline Parameter & $\rho^{\text {tot }}\left[\mathrm{t} / \mathrm{m}^{3}\right]$ & $E_{u}[\mathrm{kPa}]$ & $v_{u}[-]$ & $\Phi_{u}\left[^{\circ}\right]$ & $\psi\left[^{\circ}\right]$ & $c_{u}[\mathrm{kPa}]$ & $K_{0}[-]$ & $\sigma_{t}[\mathrm{kPa}]$ \\
\hline Value & 2.2 & 2500 & 0.49 & 0 & 0 & 80.0 & 1.0 & 1.0 \\
\hline
\end{tabular}


Table 2. Material parameters for coupled pore fluid diffusion and stress analysis (MCC model)

\begin{tabular}{|c|c|c|c|c|c|c|c|c|c|}
\hline Parameter & $\rho^{\prime}\left[\mathrm{t} / \mathrm{m}^{3}\right]$ & $G^{\prime}[\mathrm{kPa}]$ & $e_{0}[-]$ & $\kappa[-]$ & $\lambda[-]$ & $M[-]$ & $\rho_{w}[\mathrm{kPa}]$ & $K_{0}[-]$ & $k[\mathrm{~m} / \mathrm{s}]$ \\
\hline Value & 1.2 & 838.926 & 0.65 & 0.08 & 1.0 & 0.835 & 1.0 & 1.0 & $10^{-5}$ \\
\hline
\end{tabular}

The stress paths in MCC model during undrained shearing is presented in Fig. 2. It is worth noting that it is an example of undrained shearing when the initial state of soil is on the dry side of critical state line. Full consideration of this problem is presented in the Appendix. As can be seen, the crucial parameter is the preconsolidation pressure $p_{c}^{\prime}$. Let us assume all parameters, including stress ratio $M$, initial void ratio $e_{0}$, logarithmic elastic modulus $\kappa$ and logarithmic plastic modulus $\lambda$ as constant values. Hence, only the preconsolidation pressure $p_{c}^{\prime}$ will vary in accordance with the initial in-situ stress. The relation will be proceed after equation

$$
p_{c}^{\prime}=\left(\frac{4 c_{u}}{M} \cdot\left(2 p_{0}^{\prime}\right)^{-\kappa / \lambda}\right)^{\frac{1}{1-\kappa / \lambda}}
$$

where $p_{c}^{\prime}$ - pre-consolidation pressure $[\mathrm{kPa}], c_{u}$ - undrained shear strength $[\mathrm{kPa}], M-$ stress ration [-], $p_{0}^{\prime}-$ initial in-situ mean stress $[\mathrm{kPa}], \kappa$ - logarithmic elastic modulus $[-], \lambda-$ logarithmic plastic modulus $[-]$.

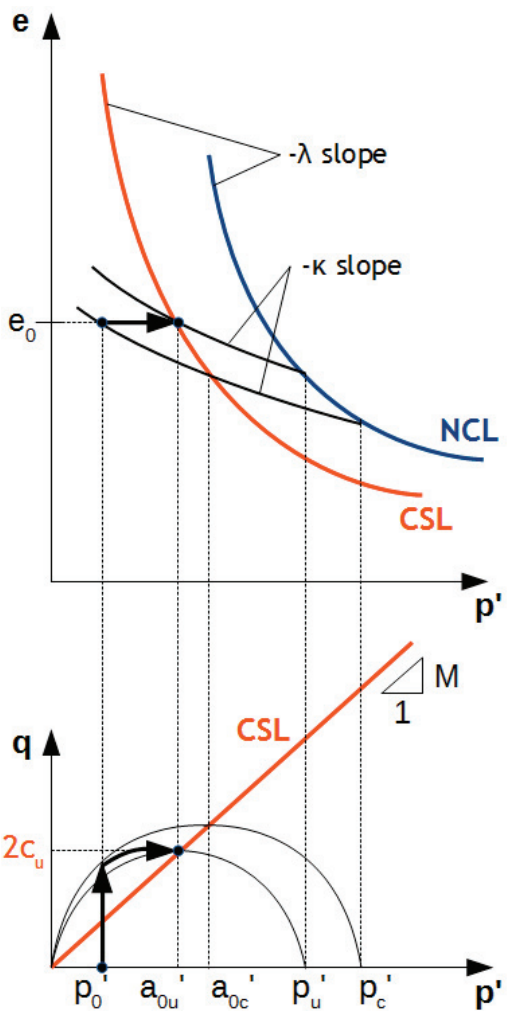

Fig. 2. Stress path for undrained shearing using Modified Cam-Clay model (notation is presented at the end of the article)
Consequently, the preconsolidation pressure $p_{c}^{\prime}$ will be varying with depth, as is shown in Fig. 3. To confirm the presented conversion between Tresca and MCC plasticity some unconsolidated undrained triaxial numerical tests were performed. The results are summarised in Fig. 4, where a relatively good agreement was achieved. The observed discrepancies depend on plastic flow time. When initial plasticity surface is reached quickly, long term plastic flow occurs. During this process small values of plastic strains are generated. The yield plastic surface is a little bit smaller than assumed in derivation of equation (6) with exponential form of hardening law, as it is implemented in Abaqus. The best accuracy is obtained when the in-situ pressure $p_{0}^{\prime}$ is almost equal to $a_{0 u}^{\prime}$ (see Fig. 2). For ideal situation, the MCC solution will be covered by Tresca solution. For simplicity, the uniform MCC model for the entire soil domain was used in this paper, with parameters shown in Table 2.

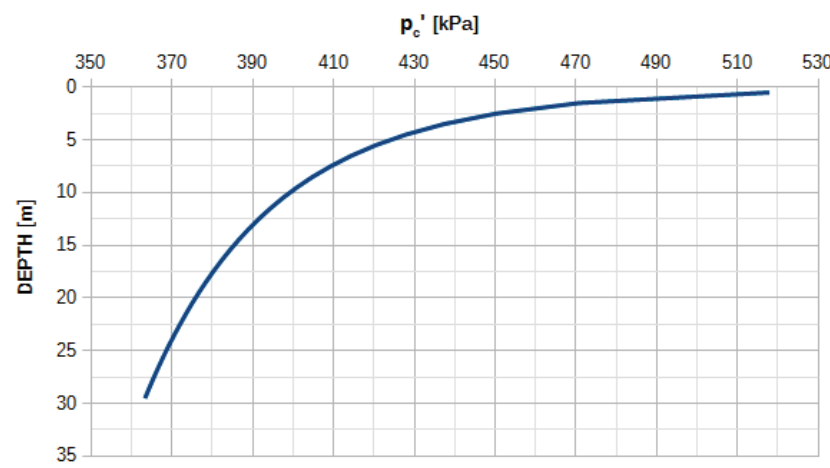

Fig. 3. Variation of preconsolidation pressure with depth for undrained shear strength assumed constant in the soil profile

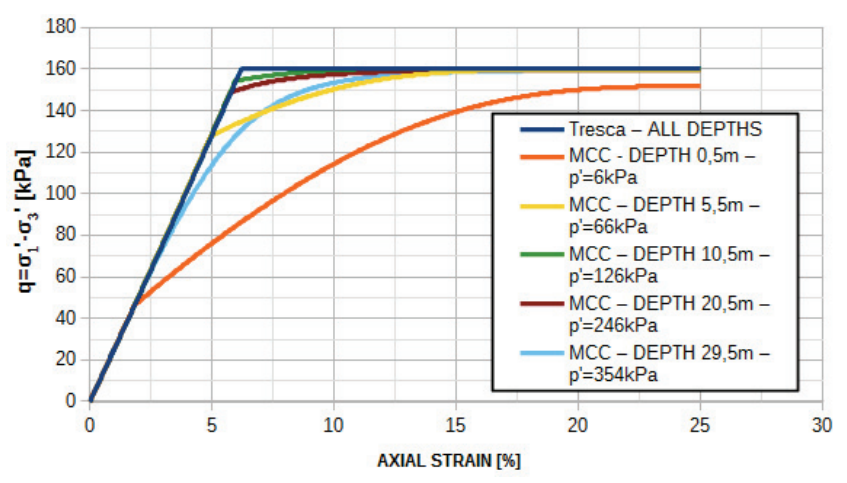

Fig. 4. Numerical triaxial UU tests for the Tresca model and correlated MCC model 
The earth pressure at rest coefficient was assumed to be equal to 1.0. This is a safe assumption because it gives the uniform distribution of pressure in all dimensions.

\section{DEVELOPING THE NUMERICAL MODELS}

The geometry of the models was the same in the two approaches presented. The models were axisymmetric with dimensions and boundary conditions presented in Fig. 5. The soil was modelled as a deformable body while the pile was assumed to be a rigid one. The pile was pre-installed in the soil at a depth of $0.5 \mathrm{~m}$ to avoid distortions of finite elements at the beginning of jacking. The process of installation was modelled using so-called zipper-technique, which was developed by Mabsout and Tassoulas (1994). In this technique, a small diameter tube supports the soil domain, as can be seen in Fig. 5. During jacking the pile slides after the tube and pushes soil outwards. The tube has diameter of $1 \mathrm{~mm}$ and frictionless contact with the soil. Contact between the pile shaft and the soil is modelled using finite sliding technique and Coulomb friction model with coefficient of friction equal to 0.231 . Due to numerical problems in the coupled pore fluid diffusion and stress analysis the contact between the pile toe and the soil was assumed to be frictionless, as was mentioned in section 2.4. The pile was jacking with velocity of $1 \mathrm{~cm} / \mathrm{s}$ to the depth of $8.0 \mathrm{~m}$.

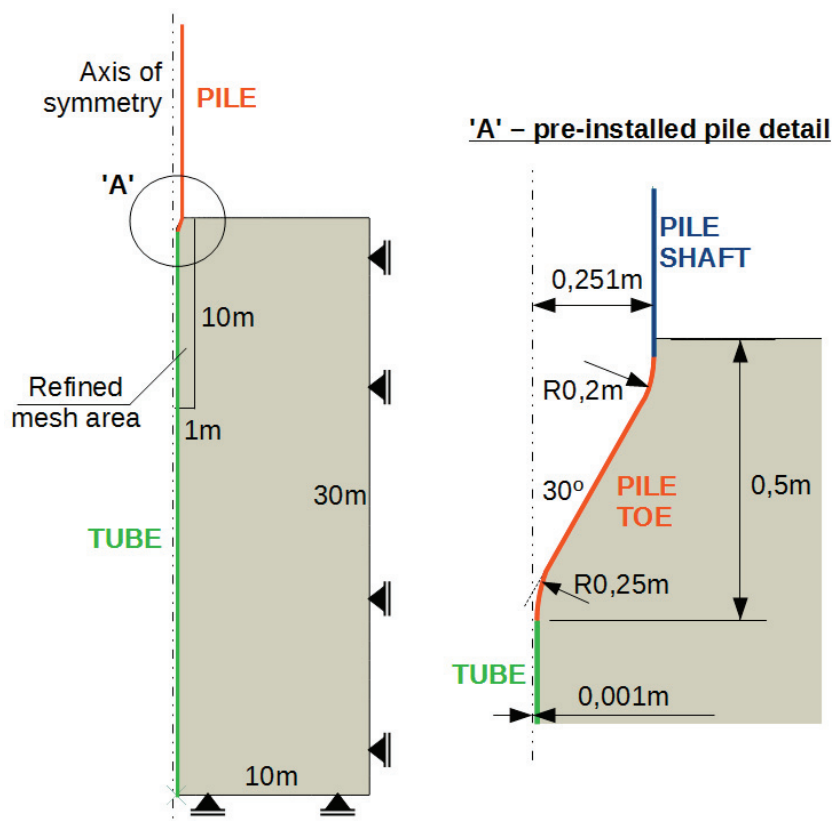

Fig. 5. Geometry and boundary conditions for undrained pile jacking simulation

\subsection{ARBITRARY \\ LAGRANGIAN-EULERIAN APPROACH}

The numerical model consists of 28803 elements with minimum element size of $2.5 \times 2.5 \mathrm{~cm}$ in the refined mesh area. The quadratic, 4 nodded, linear elements with reduced integration were used. Due to the large number of increments the analysis was conducted with double precision.

\subsection{COUPLED PORE FLUID DIFFUSION AND STRESS APPROACH}

The numerical model consists of 210883 elements with minimum size $1.0 \times 0.5 \mathrm{~cm}$ in the refined mesh area. Thirty layers of soil were assumed due to different preconsolidation pressures. The quadratic, second order elements with reduced integration were used. Although, when contact problems are involved, the first order elements are better suited, the second order elements perform better where stress concentration occurs (Dassault Systémes, 2013). Here, some compromise has to be made. As the pile jacking problem is related with large concentrated stresses the second order elements have to be used. However, the contact problem concerns mainly three-dimensional, second order elements, so in the axisymmetric model this issue does not occur. The value of permeability coefficient was assumed as $10^{-5} \mathrm{~m} / \mathrm{s}$, as stated in Table 2 . Using equation (4) and effective soil parameters the minimum usable time increment was calculated as varying from $0.22222 \mathrm{~s}$ to $0.00094 \mathrm{~s}$. During the analysis the time increments were monitored and settled in the range from $0.5 \mathrm{~s}$ to $0.00195 \mathrm{~s}$, which is higher than the estimated interval. Hence, no "overshoot" in the pore fluid pressure calculation is expected.

\section{RESULTS AND INTERPRETATION}

The most important aspect of the pile jacking is the determination of base resistance (see Fig. 6). A good agreement between the total and the effective stress analysis is achieved. The rapid increase of toe resistance to the value of $450 \mathrm{kPa}$ is observed just after start of jacking. Then a slight increase with penetration depth appears. The averaged unit shaft resistance is plotted in Fig. 7. Here, a good agreement is also obtained. The shaft resistance is mobilized more smoothly and it is about $10 \%$ of the base resistance value. 


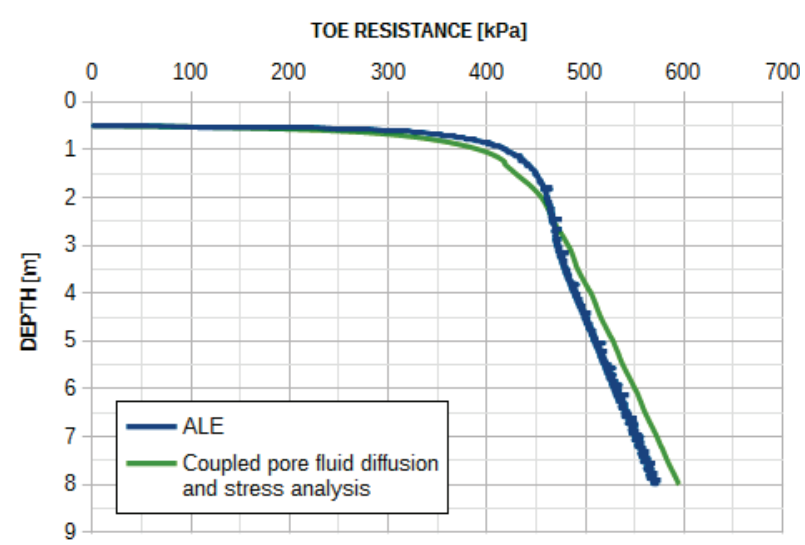

Fig. 6. Pile toe resistance during undrained jacking

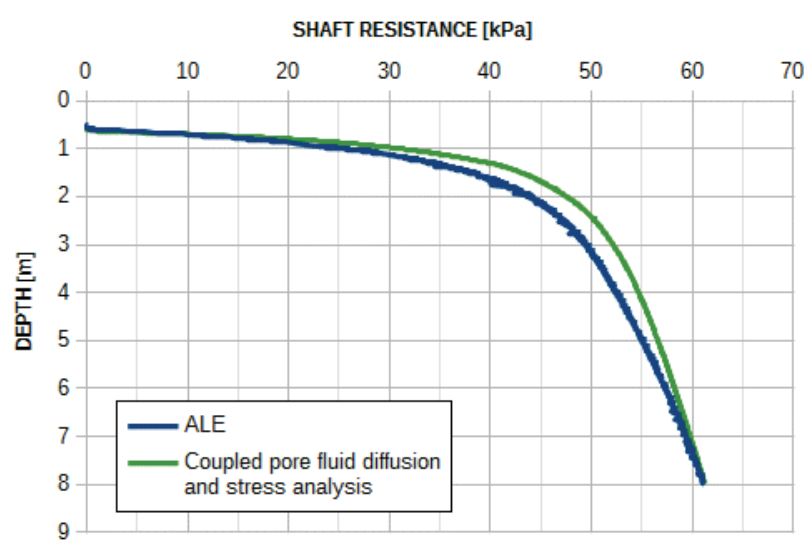

Fig. 7. Pile shaft resistance during undrained jacking

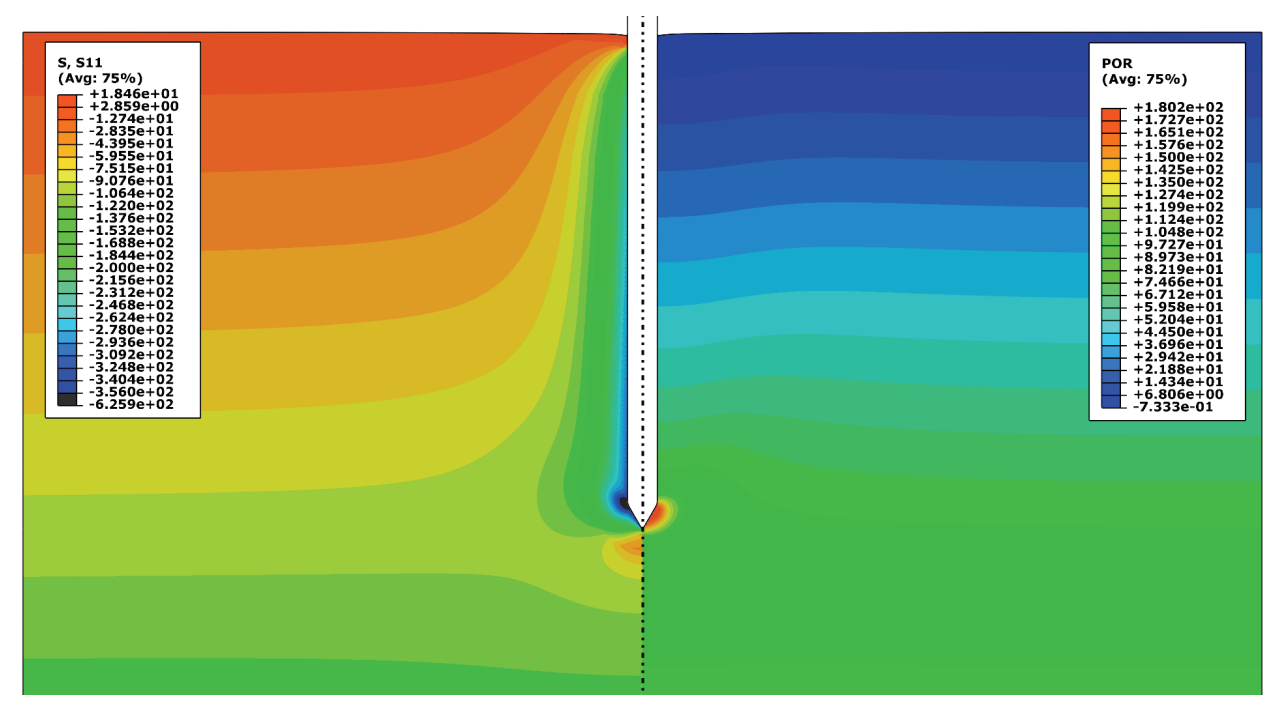

(a)

(b)

Fig. 8. Distribution of (a) radial effective stresses and (b) pore water pressures after pile installation

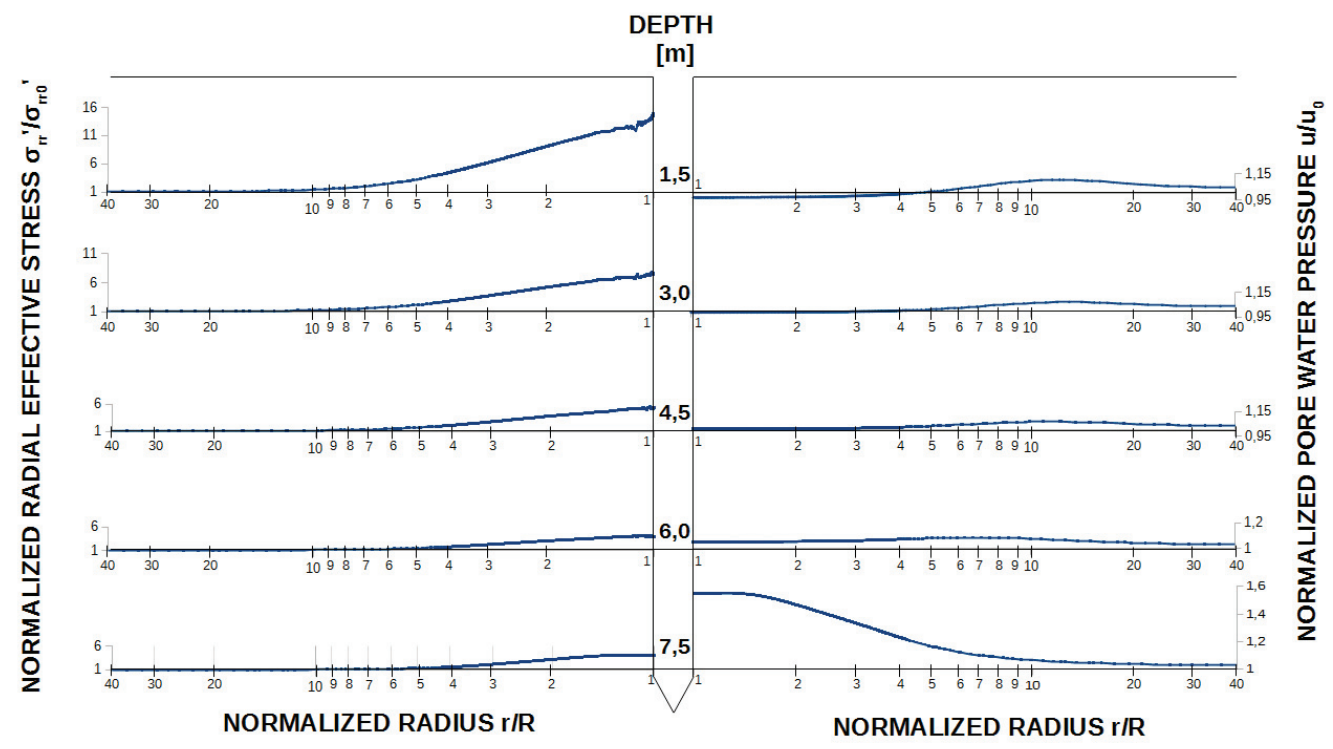

Fig. 9. Distribution of normalized radial effective stresses and normalized pore water pressures after pile installation for five depths 


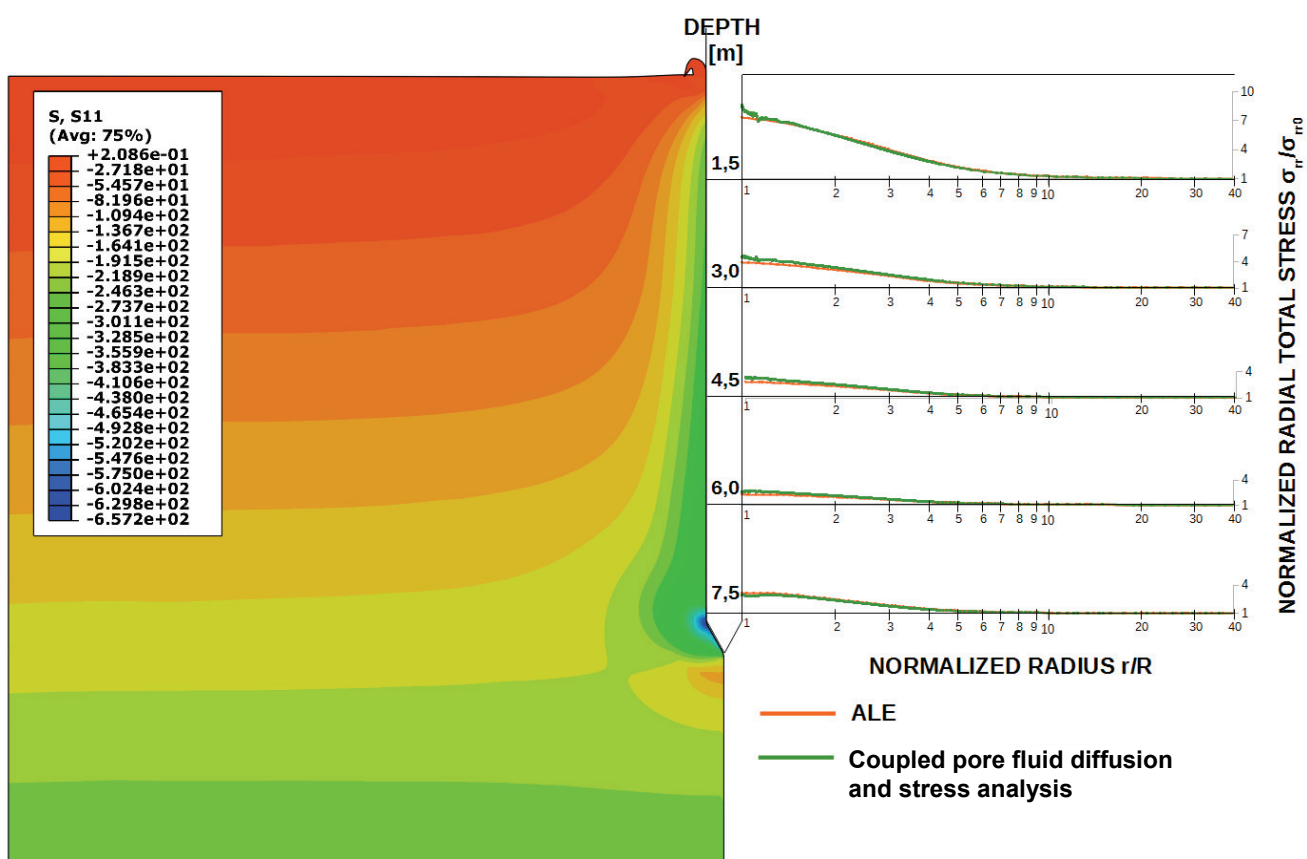

(a)

(b)

Fig. 10. Distribution of (a) radial total stresses and (b) normalized radial total stresses for five depths after pile installation

The radial effective stresses and pore water pressures distribution are presented in Fig. 8. Detailed results for five depths are shown in Fig. 9. The area affected by the pile installation can be estimated as 10 pile diameters wide. The largest generation of the pore water pressures is located near the pile tip. Upwards the shaft the pore pressure is not significantly different than the hydrostatic values. This mechanism implies the suction on the pile shaft surface. Similar effect has been observed in the field tests in high OCR clays (Bond and Jardine, 1991).

The largest accumulation of radial effective stresses is located around the pile toe. A significant increase is also noted in the pile shaft area. Similar results were also observed in other numerical studies (e.g., Zhou et al., 2013; Hamann et al., 2015). The biggest normalized gain in radial effective stress, nearby 16 times the geostatic stresses is observed near the soil surface and it decreases to 4 near the pile toe. The increase of radial effective stresses is not directly related to the undrained soil strength or other geotechnical parameter, but it depends on the failure mechanism related to pile diameter and pile toe resistance.

The total stresses achieved in both analyses are quite similar and approve the correctness of the two solutions. The map of total stresses from ALE model is presented in Fig. 10a. A comparison between ALE analysis and coupled pore fluid diffusion and stress analysis is summarized in Fig. 10b. The total stress increase of 10 times the initial value is situated near the soil surface. It can be seen that the radial total stresses calculated from the effective stress analysis are generally a little bit higher than the values obtained from total stress analysis. This is due to the combined effect of the very beginning of consolidation, which surly takes place during 750 seconds of jacking and a moderate mesh distortion near the pile surface.

\section{VERIFICATION OF NUMERICAL ANALYSIS}

The presented analyses are only pure numerical studies. The energy plots can be used to verify this kind of calculation. The FEM solution is based on energy conservation. Checking different types of energy allows us to study what is happening in the model as well as how accurate the obtained results are. This method of verification is rather dedicated to the explicit formulation, but can also be useful in implicit method. The total energy, the external work and kinetic energy for ALE analysis are plotted in Fig. 11. The change of total energy is $0.05 \%$, which is small and much lower than $1 \%$ suggested for numerically correct solution. The kinetic energy represents $13 \%$ of external work at the beginning of installation. This value is dropping to almost zero with large deforma- 
tion. Hence, the pile jacking process can be assigned as a quasi-static problem.

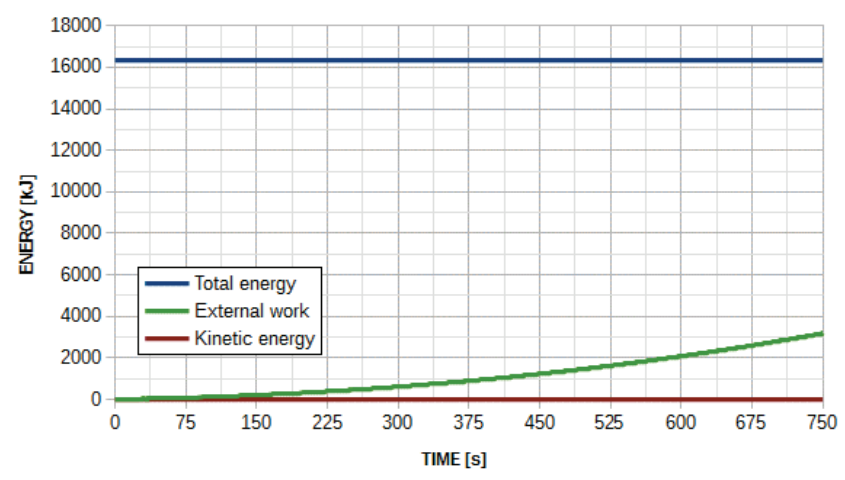

Fig. 11. Energy output for ALE analysis

Total energy change for the coupled pore fluid diffusion and stress analysis is a little bit higher and approaches $0.74 \%$, but still does not exceed $1 \%$. It is worth mentioning that total energy slightly decreases here.

\section{CONCLUSIONS}

ALE formulation in default implementation in Abaqus allows us only to model single-phase material and enforces total stress analysis. On the other hand, the coupled pore fluid and stress analysis with updated Lagrangian formulation enables two-phase material use with effective stress analysis. The analysis performed in this study has shown that a good agreement between both solutions could be achieved. ALE formulation is better suited for large deformation problems and it gives more accurate results. ALE method allows for unrestricted pile-soil contact modelling, which does not influence the computing process. Updated Lagrangian formulation used in the coupled pore fluid diffusion and stress analysis can be applicable as well. However, frictionless interaction between the pile toe and soil needs to be modelled.

The results of the analysis are in agreement with other numerical and field studies. It was found that the zone affected by the pile installation is five pile diameters wide. The ratio between pile shaft resistance and pile toe resistance is around 0.1 , which is close to the results observed for typical CPT soundings or model piles installation in OC clays. The distribution of the radial effective stress after installation is a function of the pile set-up. The important radial stress increase observed near soil surface decays with penetration depth. The highest pore water pressure is generated near pile toe. The pore water pressure distribution on the pile shaft is more complex with some local suction recorded. This distribution is influenced by the effect of pile toe and the beginning of consolidation process.

\section{NOTATION}

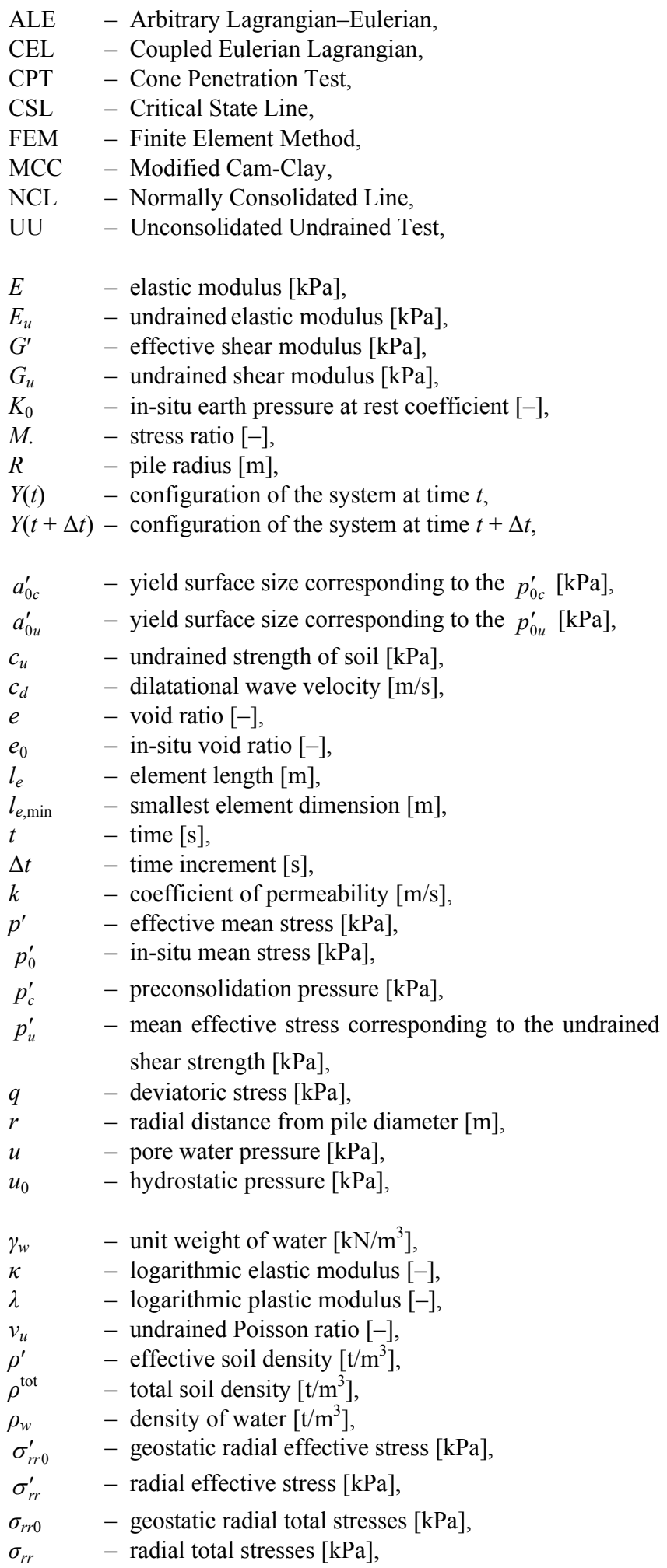


$\sigma_{t} \quad-$ tension cut-off $[\mathrm{kPa}]$,

$\Phi_{u} \quad$ - undrained angle of internal friction $\left[{ }^{\circ}\right]$,

$\psi \quad$ - dilation angle $\left[{ }^{\circ}\right]$.

\section{ACKNOWLEDGEMENTS}

The calculations were carried out at the Academic Computer Centre in Gdańsk (CI TASK).

\section{REFERENCES}

[1] ATKINSON J., The mechanics of soils and foundations, CRC Press, 2007.

[2] Bienen B., QIU G., Pucker T., CPT correlation developed from numerical analysis to predict jack-up foundation penetration into sand overlying clay, Ocean Engineering, 2015, 108, 216-226, DOI: 10.1016/j.oceaneng.2015.08.009.

[3] BOND A.J., JARDINE R.J., Effects of installing displacement piles in a high OCR clay, Geotechnique, 1991, 41(3), 341-363. DOI: 10.1680/geot.1991.41.3.341.

[4] Cummings A.E., Kerkhoff G.O., Peck R.B., Effect of driving piles into soft clay, Transactions of the American Society of Civil Engineers, 1950, 115(1), 275-285.

[5] DAI Z.H., QIN Z.Z., Numerical and theoretical verification of modified cam-clay model and discussion on its problems, Journal of Central South University, 2013, 20, 3305-3313, DOI: $10.1007 / \mathrm{s} 11771-013-1854-7$.

[6] Dassault Systémes, 2013, Abaqus 6.13 Analysis User's Guide, Dassault Systèmes.

[7] Donea J.H., Huerta A., Ponthot J.-Ph., RodrígueZFERRAn A., Arbitrary Lagrangian-Eulerian Methods, [in:] Encyclopedia of Computational Mechanic, Vol. 1. Fundamentals, John Wiley \& Sons, Ltd., 2004, 413-437, DOI: 10.1002/0470091355.ecm009.

[8] Hamann T., QIU G., Grabe J., Application of a Coupled Eulerian-Lagrangian approach on pile installation problems under partially drained conditions, Computers and Geotechnics, 2015, 63, 279-290, DOI: 10.1016/j.compgeo.2014.10.006.

[9] Komurka V.E., Wagner A.B., Edil T.B., A Review of Pile Set-Up, Proc., 51st Annual Geotechnical Engineering Conference, 2003.

[10] Mabsout M.E., Tassoulas J.L., A finite element model for the simulation of pile driving, International Journal for numerical methods in Engineering, 1994, 37(2), 257-278, DOI: 10.1002/nme. 1620370206 .

[11] NoH W.F., CEL: a time-dependent, two-space-dimensional, coupled Eulerian-Lagrangian code. Lawrence Radiation Lab., Univ. of California, Livermore, 1963.

[12] PotTs D.M., ZdRAVKović L., Finite element analysis in geotechnical engineering: theory, Vol. 1, Thomas Telford, 1999, DOI: 10.1680/feaiget.27534.

[13] Tho K.K., Leung C.F., Chow Y.K., Swaddiwudhipong S., Eulerian finite element simulation of spudcan-pile interaction, Canadian Geotechnical Journal, 2013, 50(6), 595-608, DOI: $10.1139 /$ cgj-2012-0288.

[14] Vermeer P.A., VerRUiJT A., An accuracy condition for consolidation by finite elements, International Journal for Numerical and Analytical Methods in Geomechanics, 1981, 5(1), 1-14. DOI: 10.1002/nag.1610050103

[15] Yi J.T., ZhaO B., Li Y.P., YANG Y., LeE F.H., GoH S.H., ZHANG X.Y., WU J.F., Post-installation pore-pressure changes around spudcan and long-term spudcan behaviour in soft clay, Computers and Geotechnics, 2014, 56, 133-147, DOI: 10.1016/j.compgeo.2013.11.007.

[16] Zhou T.Q., Tan F., Li C., Numerical Analysis for Excess Pore Pressure Dissipation Process for Pressed Pile Installation, Applied Mechanics and Materials, 2013, Vol. 405, 133-137, DOI: 10.4028/www.scientific.net/AMM.405-408.133.

\section{APPENDIX}

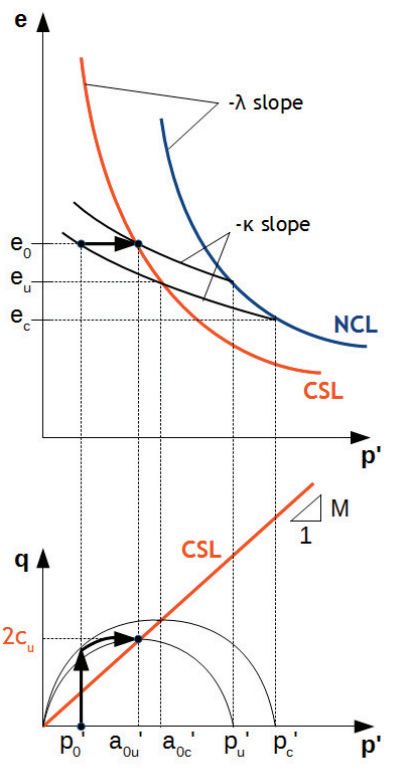

(a)

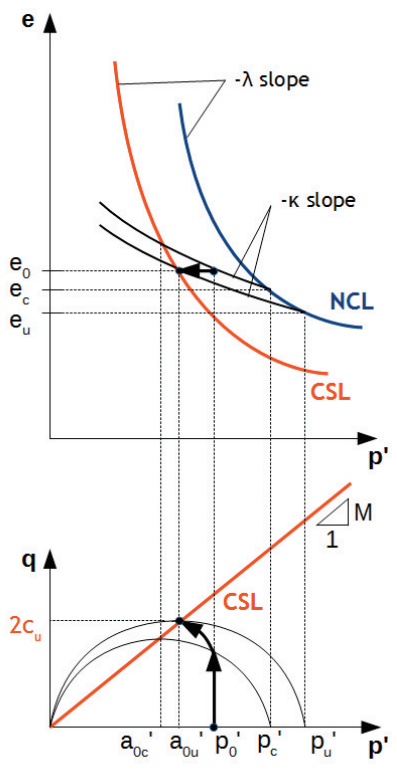

(b)
Fig. A1. Stress paths during undrained shearing when initial state of soil is on (a) dry side of the critical line,

(b) wet side of the critical line

The fitting between the undrained shear strength of the soil and MCC model can be obtained by the assumption of constant void ratio during shearing. The initial state of the soil can be on dry or wet side of critical line, so two stress paths are possible during undrained shearing, as is presented in Fig. A1. For the dry side the following equations can be written

$$
\begin{aligned}
& e_{0}-e_{c}=\kappa \cdot \ln \left(\frac{p_{c}^{\prime}}{p_{0}^{\prime}}\right), \\
& e_{0}-e_{u}=\kappa \cdot \ln \left(\frac{p_{u}^{\prime}}{a_{0 u}^{\prime}}\right), \\
& e_{u}-e_{c}=\lambda \cdot \ln \left(\frac{p_{c}^{\prime}}{p_{u}^{\prime}}\right) .
\end{aligned}
$$

Using the assumption of yield surface size in MCC model one can write 


$$
a_{0 u}=\frac{1}{2} p_{u}
$$

Consequently, equation (A2) can be rewritten as

$$
e_{0}-e_{u}=\kappa \cdot \ln (2) \text {. }
$$

Combining equation (A1), (A3) and (A5) we get

$$
\kappa \cdot \ln \left(\frac{p_{c}^{\prime}}{p_{0}^{\prime}}\right)=\lambda \cdot \ln \left(\frac{p_{c}^{\prime}}{p_{u}^{\prime}}\right)+\kappa \cdot \ln (2) .
$$

Equation (A6) can be transformed to the following form

$$
\left(\frac{p_{c}^{\prime}}{2 p_{0}^{\prime}}\right)^{\kappa / \lambda}=\frac{p_{c}^{\prime}}{p_{u}^{\prime}}
$$

From definition of stress ratio $M$ (Fig. 6), we know that

$$
M=\frac{2 c_{u}}{a_{0 u}} .
$$

Combining equation (A8) and equation (A4) we get

$$
p_{u}=\frac{4 c_{u}}{M}
$$

From equation (A7) and equation (A9) we can derive equation (6)

$$
p_{c}^{\prime}=\left(\frac{4 c_{u}}{M} \cdot\left(2 p_{0}^{\prime}\right)^{-\kappa / \lambda}\right)^{\frac{1}{1-\kappa / \lambda}} .
$$

Similar consideration can be made for the wet side of critical line. We can write the following equations

$$
\begin{aligned}
& e_{0}-e_{c}=\kappa \cdot \ln \left(\frac{p_{c}^{\prime}}{p_{0}^{\prime}}\right), \\
& e_{0}-e_{u}=\kappa \cdot \ln \left(\frac{p_{u}^{\prime}}{a_{0 u}^{\prime}}\right), \\
& e_{c}-e_{u}=\lambda \cdot \ln \left(\frac{p_{u}^{\prime}}{p_{c}^{\prime}}\right) .
\end{aligned}
$$

We can notice that equations (A1) and (A11) as well as equations (A2) and (A12) are the same. Let us transform equation (A13) into the following form

$$
e_{u}-e_{c}=-\lambda \cdot \ln \left(\frac{p_{u}^{\prime}}{p_{c}^{\prime}}\right) .
$$

Thus, using the power low of logarithm we get

$$
e_{u}-e_{c}=\lambda \cdot \ln \left(\frac{p_{c}^{\prime}}{p_{u}^{\prime}}\right)
$$

Hence, equation (A15) is the same as equation (A3) and the equation for the preconsolidation pressure is the same for wet and dry side of the critical line and it is described by equation (A15) and (6). 